\title{
Study on Performance of Foamed Ceramics in Soot Trapping
}

\author{
Zhilin Liu \\ Department of Engineering Mechanics \\ Agricultural and Animal Husbandry College of Tibet \\ University \\ Linzhi, China \\ Linlin0324ok@126.com
}

\author{
Jun Bai \\ Department of Energy and Power Engineering \\ Agricultural and Animal Husbandry College of Tibet \\ University \\ Linzhi, China
}

\begin{abstract}
In order to obtain the foamed ceramic trapping efficiency on soot, a soot trapping installation was designed, made test scheme of soot trapping according to ELR test cycle. Through the analysis of test data, the conclusions are as follows: the trapping performance of 25 PPI foamed ceramics is obviously better than the trapping performance of $10 \mathrm{PPI}$ foamed ceramics; the trapping performance of foamed ceramics will be continually improved with the increase of the thickness; the increase of the space of the foamed ceramics is conducive to improve the efficiency of soot trapping.
\end{abstract}

Keywords- foamed ceramics; particle; soot trapping

\section{INTRODUCTION}

With the rapid development of automobile industry, the number of vehicles is expanding. The vehicle exhaust has become the first pollutant of urban air [1] [2]. With the continuous improvement of environmental quality requirements, the control of urban air pollutant is increasingly strict. Many cities have implemented high emission control standards. The diesel engine has been widely used in various fields because it is an internalcombustion engine of good economy, low $\mathrm{CO}$ and $\mathrm{HC}$ emission. However, lots of particles will be produced while the diesel engine is working. For the methods to control the particles, development of soot particle trapping installation to reduce the particle emission has become a key, in addition to improve the internal structure of diesel engine.

\section{FREQUENTLY-USED MATERIALS FOR SOOT TRAPPING}

Frequently-used materials for soot trapping include ceramic fiber, honeycomb ceramics, foamed ceramics, metal base material and composites.

\section{A. Ceramic Fiber}

Ceramic fiber felt has high surface area, the fiber surface in the filter of felt is all the effective filtration area, the filter efficiency can up to $95 \%$. However, there are some weaknesses such as low heat resistance, complicated production process and easy to damage [3].

\section{B. Honeycomb Ceramics}

Honeycomb ceramics has many advantages such as high filter efficiency and structural strength. But its weakness is high cost, and the characteristic of anisotropy could cause partial melting or rupture easily while the ceramics is heating uneven [4].

\section{Foamed Ceramics}

Foamed ceramics has been used to filter the particles in the exhaust of diesel engine, because compared with honeycomb ceramic, foamed ceramics has good plasticity and high porosity (80\%-90\%), and the hollow is very tortuous. The isotropy while be heated makes foamed ceramics has better thermal stability. However, the filter efficiency of foamed ceramics is not very good.

\section{Metal Base Material}

Metal base material has advantages of high strength and toughness, good thermal conductivity. At present, most studies focus on foamed alloy and metal fiber felt.

\section{E. Composites}

Complex filter material has been considered because ceramics based material and metal base material have inevitable defects. At present, studies and applications are mainly focus on fiber felt.

\section{THE PROPERTIES AND SOOT TRAPPING MECHANISM OF FOAMED CERAMICS}

\section{A. The Characteristic Parameter of Foamed Ceramics [5]}

\section{1) Strength}

The flexural strength of foamed ceramic can refer to the following formula [6]:

$$
R=3 P \cdot L /\left(2 b \cdot h^{2}\right)
$$

In the formula, $R$ is the flexural strength of sample, $P$ is the load on the sample, $L$ is the distance among pivots, $b$ is width of sample, $h$ is the thickness of sample.

\section{2) Thermal-shock stability}

Thermal-shock stability is the comprehensive inflection to mechanical properties and thermal properties of foamed ceramics. Therefore, the factors of thermal-shock properties of foamed ceramics is manifested in the respects of thermal parameters and mechanical parameters which includes coefficient of linear extensibility thermal conductivity, elastic modulus, cracked surface energy [7].

3) The average pore diameter

The pore shape of foamed ceramics is very complex and irregular, so the average pore diameter is often to express 
the pore diameter of foamed ceramics. The calculation method is as follow:

$$
d=\frac{4 \sigma \cdot \cos \theta}{p}
$$

In the formula, $d$ is capillary diameter, $\sigma$ is the surface tension of fluid, $p$ is the press needed to make fluid pass through capillary, $\theta$ is wetting angle of fluid material.

4) Porosity

Porosity is the ratio of pore volume and total volume. The calculation method is as follow:

$$
\varepsilon=\frac{V_{k x}}{V}
$$

In the formula, $V_{k x}$ is pore volume, $V$ is total volume.

The porosity of foamed ceramic can reach $75 \% \sim 90 \%$, which porosity is the highest of the porous ceramics. For example, the porosity of honeycomb ceramic is about $60 \%$, and the porosity of ceramic particles sintered body is in $30 \% \sim 50 \%$.

\section{5) Penetration ability}

The penetration ability of foamed ceramics is the ability of fluid pass through the material, it is often expressed by permeability. As the collection of capillaries, the law of fluid passing through capillary can be described by Poisewille method:

$$
v=\frac{\pi d^{4} \Delta p}{128 \alpha \cdot \mu \cdot L}
$$

In the formula, $v$ is the flow of fluid through capillary, $\triangle p$ is differential pressure of both sides of material, $L$ is the thickness of material, $\mu$ is viscosity of fluid, $\alpha$ is the bending degree of pore.

\section{B. The Soot Trapping Mechanism of Foamed Ceramics}

The soot trapping is realized by particle filter, its mechanism is often divided into two basic types --- deep bed filtration and cake filtration.

\section{1) Deep bed filtration}

Because the filter media has stereo pore structure, so it can collect solid particles smaller than pores, it also can collect solid particles much smaller than pores in the deep of filter media. Its mechanism [8] [9] is as shown in figure 1.
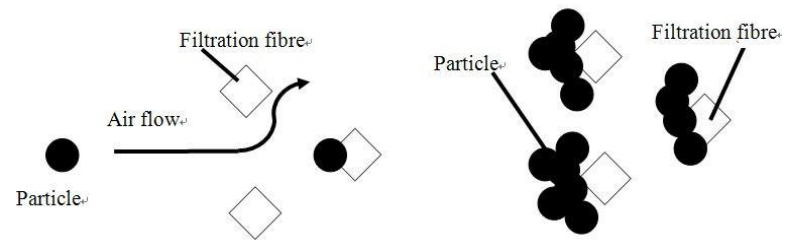

Figure 1. Deep bed filtration mechanism [8]

Deep bed filtration has complex mixing mechanism. It mainly includes diffusion, interception and inertial impaction. Under the inertial force or the action of Brownian motion, particle contacts with the pore wall and adhere to the wall, and then the particles will adsorption together under the action of Van der Waals forces or other surface forces. Therefore, the purpose of adsorbing particles has been realized.

\section{2) Cake filtration}

Cake filtration is that the filter cake will be produced with the progress of filtration. The filtration cake itself can play the role of filter media. The solid particles is intercepted by filtration cake, but fluid could pass through filtration cake and media framework, causing the filtration cake continued thickening, therefore, the particles in fluid will dwindle.

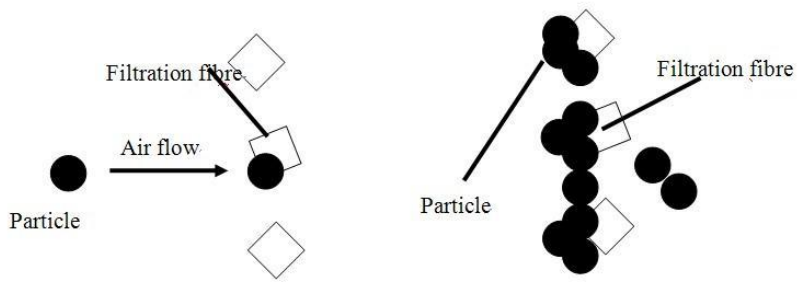

Figure 2. Cake filtration mechanism [8]

\section{SOOT TRAPPING TESTS}

\section{A. The Tested Foamed Ceramics and Soot Trapping Installations}

\section{1) The tested foamed ceramics}

The tested material are 10PPI (Part per inch) SIC porous foamed ceramics and 25PPI $\mathrm{Al}_{2} \mathrm{O}_{3}$ porous foamed ceramics, which are produced by Guangdong Foshan Ceramic Group. The high temperatures of these foamed ceramics can withstand are $1500{ }^{\circ} \mathrm{C}$ and $1200{ }^{\circ} \mathrm{C}$ respectively. Shapes of all the material samples are cylindrical. The diameter, thickness each piece and porosity of 10PPI foamed ceramics tested are $160 \mathrm{~mm}, 21 \mathrm{~mm}$ and 0.8107 respectively, and the diameter, thickness each piece and porosity of 25PPI foamed ceramics tested are $160 \mathrm{~mm}, 23 \mathrm{~mm}$ and 0.8834 respectively. In the tests, using a combination of two or more pieces foamed ceramics in order to obtain greater thickness, because the thicknesses of foamed ceramics produced by factory are fixed.

\section{2) Soot trapping installations}

The soot trapping installations as shown in figure 3 . The exhaust of diesel engine enter into soot trapper from left side of the pipe, exhaust is discharged from right side of the pipe after pass through the foamed ceramics. In the process of exhaust passing through foamed ceramics, the soot trapper will trap soot particles by mechanisms of diffusion trapping and inertial collision. The soot particles trapped are adsorbed on the foamed ceramics. 


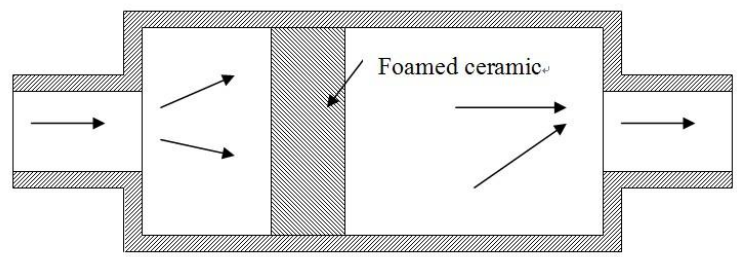

Figure 3. Diagram of soot trapping installations

In addition, in order to facilitate the replacement of different types of foamed ceramics and its combination, the soot trapping installation tested is designed to detachable two-section-type structure. The trapping performance of different types of foamed ceramics and its combination can be obtained through using the smoke meter measure the smoke values of both the before and after sides of the soot trapper [10].

\section{B. Soot Trapping Tests Schemes}

The running of engine should according to the requirements of ELR test cycle. Before the exhaust enter into the soot trapper, using the smoke meter measure the smoke values of exhaust, and then measure the smoke values again after the exhaust has passed through the soot trapper [11][12]. Recording the relevant data by AVL smoke meters, and then trapping calculate the efficiency of soot trapper.

Tests will respectively research the trapping performance of $42 \mathrm{~mm}$ thickness, 10 PPI SIC porous foamed ceramics and $46 \mathrm{~mm}$ thickness, 25PPI $\mathrm{Al}_{2} \mathrm{O}_{3}$ porous foamed ceramics, selecting the better one after comparison of their trapping performance, and then do further studies through adjusting the thickness and distance.

\section{The Comparison and Analysis of Test Data}

\section{1) The test data comparison of 10 PPI and 25 PPI}

The test data comparison of 10 PPI and 25 PPI as shown in figure 4 , curve $\mathrm{A}$ is original soot emission value, curve $\mathrm{B}$ is the soot emission value after installing $42 \mathrm{~mm}$ thickness 10PPI foamed ceramic, curve $C$ is the soot emission value after installing $46 \mathrm{~mm}$ thickness 25PPI foamed ceramic. From the comparison of the test data in the figure, we can find that the effect of $42 \mathrm{~mm}$ thickness 10PPI foamed ceramic to reduce the soot emission is not very obvious. The effect of $46 \mathrm{~mm}$ thickness 25PPI foamed ceramic to reduce the soot emission is obviously better than the effect of $42 \mathrm{~mm}$ thickness 10PPI foamed ceramic to reduce the soot emission. The pore diameter of 10 PPI foamed ceramic is relatively large, so the intercepting effect of soot particles is relatively poor. However, the intercepting effect of 25PPI foamed ceramic to soot particles is better because its pore diameter is relatively smaller.

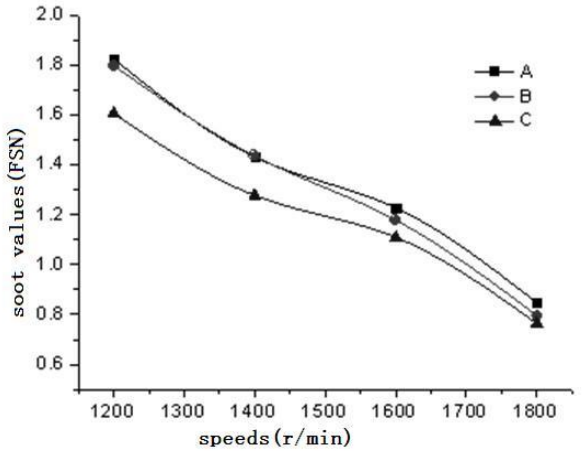

A-the original value, B-10PPI 42mm thickness, C-25PPI 46mm thickness

Figure 4. The comparison of smoke value

The comparison of trapping efficiency is shown in figure 4, curve B is the soot trapping efficiency of $42 \mathrm{~mm}$ thickness 10PPI foamed ceramic, curve $\mathrm{C}$ is the soot trapping efficiency of $46 \mathrm{~mm}$ thickness 25PPI foamed ceramic. From figure 4 we can find that the average soot trapping value of 10PPI foamed ceramic is about $3 \%$, but the average soot trapping value of 25PPI foamed ceramic is up to about $10.5 \%$. Therefore, it is obviously that the trapping performance of $46 \mathrm{~mm}$ thickness, 25 PPI foamed ceramic is much better than the trapping performance of $42 \mathrm{~mm}$ thickness, 10 PPI foamed ceramic.

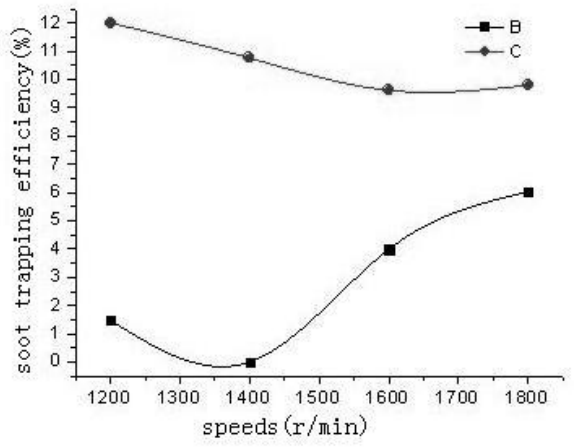

B-10PPI 42mm thickness, C-25PPI 46mm thickness

Figure 5. The comparison of trapping efficiency

\section{2) The effect of distance to 25 PPI foamed ceramic}

Installing two pieces of 25 PPI foamed ceramics in the soot trapper (the total thickness is $46 \times 2=92 \mathrm{~mm}$ ), set the distances are $25 \mathrm{~mm}$ and $100 \mathrm{~mm}$, respectively measure the soot values.

In figure 6, curve $\mathrm{A}$ is the original soot emission value, curve D is the soot emission value after installing $92 \mathrm{~mm}$ thickness $25 \mathrm{~mm}$ distance, 25PPI foamed ceramics, curve E is the soot emission value after installing $92 \mathrm{~mm}$ thickness $100 \mathrm{~mm}$ distance, 25PPI foamed ceramics. With the increase of distance between two ceramics, the trapping performance will be improved from the comparison of curve $\mathrm{D}$ and curve E. The change of distance makes the change of flow field of foamed ceramics, cause the changes of diffusion, inertial interception and trap to soot particles. Therefore, the increase of distance between two ceramics makes the overall trapping efficiency is improved obviously. 


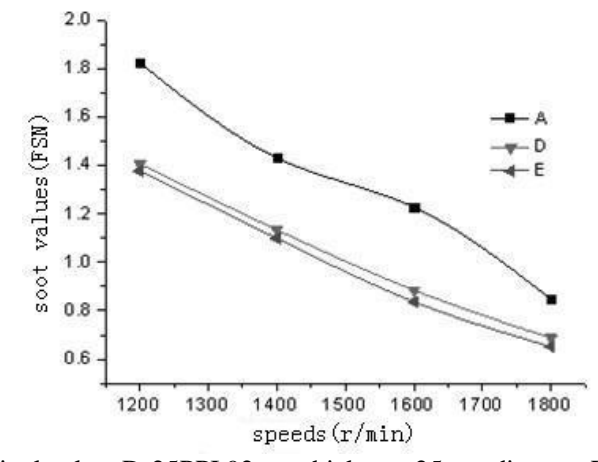

A-the original value, D-25PPI 92mm thickness $25 \mathrm{~mm}$ distance E-25PPI $92 \mathrm{~mm}$ thickness $100 \mathrm{~mm}$ distance

Figure 6. The comparison of smoke value

The trapping efficiency comparison of different distance combination of ceramics is shown in figure 7 , curve $\mathrm{D}$ is the soot trapping efficiency of $92 \mathrm{~mm}$ thickness, $25 \mathrm{~mm}$ distance, 25PPI foamed ceramics, curve $\mathrm{E}$ is the soot trapping efficiency of $92 \mathrm{~mm}$ thickness, $100 \mathrm{~mm}$ distance, 25PPI foamed ceramics. The average trapping efficiency of $25 \mathrm{~mm}$ distance is $22.61 \%$, while the average trapping efficiency of $100 \mathrm{~mm}$ distance is $25.63 \%$. It indicates that the increase of distance helps to improve the soot trapping efficiency.

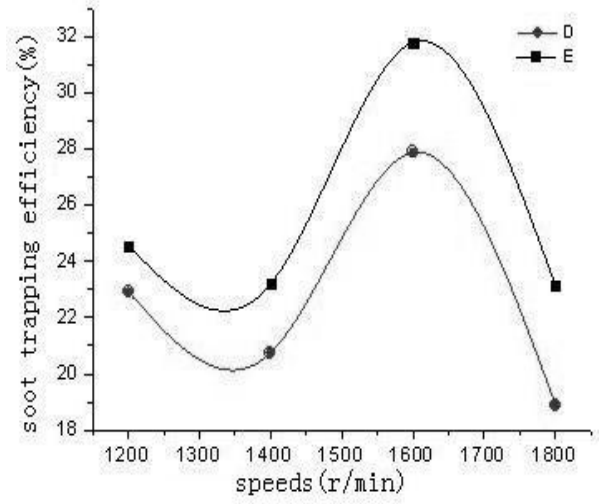

D-25PPI 92mm thickness 25mm distance E-25PPI 92mm thickness $100 \mathrm{~mm}$ distance

Figure 7. The comparison of trapping efficiency

\section{CONCLUSIONS}

1. The soot trapper based on foamed ceramics has obvious trapping function to the particles in the engine exhaust;
2. The trapping performance of $46 \mathrm{~mm}$ thickness, 25 PPI foamed ceramic is much better than the trapping performance of $42 \mathrm{~mm}$ thickness, 10 PPI foamed ceramic;

3. With the increase of thickness, interception and inertial collision of foamed ceramics to soot particles increase, so the soot trapping efficiency is improved;

4. The increase of distance helps to improve the soot trapping efficiency.

\section{ACKNOWLEDGMENT}

This article is supported by "The project of comprehensive construction to rural energy (2013) ---The construction of ecological Demostration Village to the Development and Utilization of Tibet Energy".

\section{REFERENCES}

[1] Jun Qin, Lin Lv. "Study on the route of China IV heavy-duty diesel engine technology " J. Engine information for vehicle, 2007, 5(4): pp. 4-9

[2] Hong He, Duan Weng. "Overview of technology to diesel engine emission control" J. Environmental Science, 2007, 28(6): pp.11691171.

[3] Edward Jobson. "Future Challenges in Automotive Emission Control." J. Topics in Catalysis, 2004, 28(1-4): 191-199.

[4] Masoudi M, Heibel A, Then P M. "Predicting Pressure Drop of WallFlow Diesel Particulate Filters-Theory and Experiment. "J. SAE, 2000, 2000-01-0184.

[5] Minhua Luo, "Practical technology of porous ceramics." M. Beijing: China Building Industry Press, 2006, pp: 35-36.

[6] Yang Liu, Xuejun Song. "The development situation of foamed ceramics preparation methods." J. Ceramics, 2009 (2), pp: 33-34.

[7] Yanling Dong, Weimin Wang. "Study on anti-thermal-shock property of ceramics material." J. Modern technical ceramics, 2004, 25(1), pp: 37-41.

[8] Changsong Hu. "Study on the soot trapping performance to engine exhaust and numerical simulation." D. Shanghai: Shanghai Jiaotong University, 2008, pp: 16-18, 42-45.

[9] Weiyi Wang, Qisheng Ding. "Filter medium and its selection." $M$. Beijing: China Textile Press, 2008, pp:96-99.

[10] Yuxiang Zhou, Zezhi Chen. "The design and experiment of soot trapper to diesel engine." J. Agricultural machinery Journal, 2005, 36(6), pp: 28-30.

[11] Zhixin Wang, Xuezhi Ji. "Discussion on the particles and soot in exhaust of the diesel engine." J. Diesel Engine, 2006, 28(6), pp: 1921.

[12] Chun Xu, Zizhong Ren. "Numerical simulation to soot emission of middle-speed diesel engine.” J. Diesel Engine, 2005, 27(1), pp: 24-27. 